\title{
Evaluation of age effects on doxorubicin-induced toxicity in mesenchymal stem cells
}

\author{
Ali Bashiri Dezfouli ${ }^{1}$, Ali Akbar Pourfathollah², Jamileh Salar-Amoli3*, Mohammad Khosravi ${ }^{4}$, \\ Mahin Nikogoftar-Zarif ${ }^{5}$, Mina Yazdi ${ }^{6}$, Tahereh Ali-Esfahani ${ }^{7}$
}

Received: 5 Jan 2017

Published: 17 Dec 2017

\begin{abstract}
Background: Doxorubicin, by aggregating in bone marrow, causes genotoxic effects, and thus reduces the repair ability of cells. The present study was conducted as an in vitro evaluation of age effects on the cytotoxicity induced by doxorubicin in mesenchymal stem cells (MSCs).

Methods: The MSCs of female BALB/c mice aged 1, 8, and 16 months were separated, characterized, and subsequently evaluated in cellular growth media. After 24 hours, exposure of the MSCs of the 3 groups of mice to doxorubicin $(25,50,100,200,400,800,1200$ $\mathrm{nM}$ ) and cytotoxicity were assessed, and the sublethal dose was determined using flow cytometry technique and lactate dehydrogenase $(\mathrm{LDH})$ release assay.

Results: The IC50 values determined by flow cytometry for the separated MSCs of 1 young, 8 middle- aged, and 16 old mice were and respectively. Interestingly, the results of these 2 methods in determining cytotoxicity were in agreement, and a concentration of approximately $25 \mathrm{nM}$ was considered to be the shared sublethal dose for different ages.

Conclusion: The results indicated that MSCs of middle-aged mice were more resistant to the toxic effects of the drug. Besides, MSCs separated from the old mice were the most sensitive to chemotherapy and its side effects such as disruptions of cell proliferation and viability. These disruptions can be ascribed to the alteration of function and physiological processes with age. Determining proper concentration of doxorubicin drug to destruct cancerous cells based on age and individual sensitivity can minimize the amount of toxicity.
\end{abstract}

Keywords: Doxorubicin, Mesenchymal Stem Cells, Cytotoxicity, Age

Copyright $₫$ Iran University of Medical Sciences

Cite this article as: Bashiri Dezfouli A, Pourfathollah AA, Salar-Amoli J, Khosravi M, Nikogoftar-Zarif M, Barin A, Yazdi M, Ali-Esfahani T. Evaluation of age effects on doxorubicin-induced toxicity in mesenchymal stem cells. Med J Islam Repub Iran. 2017 (17 Dec);31:98 https://doi.org/10.14196/mjiri.31.98

\section{Introduction}

Due to the growing incidence of different cancers in humans, chemotherapy has increasingly been used for their complete cure and/or prevention. Doxorubicin antibiotic (with the commercial name of Adriamycin) belongs to the group of anthracyclines, which disrupt the process of cancerous cell growth through induction of apoptosis, disruption of DNA replication, shortening of telomere length, and generation of free radicals (1-3). Cancer

Corresponding author: Dr Jamileh Salar-Amoli, jsalar@ut.ac.ir

1. Department of Basic Sciences, Faculty of Veterinary Medicine, University of Tehran, Tehran, Iran.

2. Department of Immunology, Faculty of Medical Science, Tarbiat Modares University, Tehran, Iran.

3. Department of Basic Sciences, Toxicology and Animal Poisoning Research Center, Faculty of Veterinary Medicine, University of Tehran, Tehran, Iran.

4. Department of Pathobiology, Faculty of Veterinary Medicine, Shahid Chamran University of Ahvaz, Ahvaz, Iran.

5. Blood Transfusion Research Center, High Institute for Research and Education in Transfusion Medicine, Tehran, Iran.

6. Faculty of Veterinary Medicine, University of Tehran, Tehran, Iran.

7. Toxicology and Animal Poisoning Research Center, Faculty of Veterinary Medicine, University of Tehran, Tehran, Iran. treatment mainly aims to destroy cancerous cells without damaging the normal cells in the body. Most chemical drugs such as doxorubicin have no specialized function in destruction of tumor tissues, and unfortunately they can adversely affect many normal bodily tissues and their surrounding niches $(4,5)$. On the other hand, due to the incidence of drug resistance and potential side effects of doxorubicin such as bone marrow depression and injury to the

$\uparrow$ What is "already known" in this topic:

Doxorubicin can negatively affect MSCs; many factors control the sensitivity of MSCs toward chemotherapy. Taking these factors in to consideration can be a good step toward developing therapeutic strategies.

$\rightarrow$ What this article adds:

During an in vitro study, the age factor in MSCs extracted from $\mathrm{BALB} / \mathrm{c}$ mice was considered in evaluation of doxorubicin toxicity. The 3 different age MSCs showed cytotoxic related changes, followed by treatment with doxorubicin, and the middle age group demonstrated less sensitivity. 
myocardium, the use of this drug in cancer therapy is limited. One of the main target tissues for doxorubicin drug is bone marrow, for which drug concentration after perfusion has been reported to be 200 to 500 times greater than plasma (4). This drug has cytotoxic effects on different cells of bone marrow including MSCs, as recorded in the literature $(2,6,7)$. Doxorubicin side effects are often dosedependent, and thus determining the sublethal dose of the drug for normal body cells is of great importance.

MSCs have already attracted much attention in the fields of gene therapy and regenerative medicine because of their characteristics such as ease of differentiation, rapid proliferation, and possibility of genetic handling (8). Bone marrow is a known and accessible source for these cells. Although these mesoderm-derived multipotent cells comprise a very low percentage of the nucleate cells of the bone marrow, they play a key role in supporting hematopoietic stem cells (HSCs) by their presence in the niche of the HSC cells. MSCs are capable of regulating the immune system, and varied conditions in the injured bodily tissue lead to their movement toward the injury location and their subsequent differentiation. This class of stem cells has also been differentiated from other tissues such as umbilical cord, fat tissue, and tooth pulp. Furthermore, as MSCs are a valuable cellular resource for cell therapy and regenerative processes, they are often used as a pattern for the study of bones, cartilages, muscles, and other connective tissues $(8,9)$.

Although MSCs are highly resistant to chemotherapy, changes in morphology, surface markers, and reduced differentiation are observed following treatment (10). Doxorubicin could reduce growth rate and generation of alkaline phosphatase and could also prevent synthesis of Connextion 43 and Troponin T in MSCs (2). Due to such toxic effects in more recent studies, it has been suggested that autologous graft of MSCs cannot be a suitable treatment choice to compensate for the myotoxic effects of doxorubicin (11).

As age is always an influencing factor in growth, proliferation, and metabolic activities of stem cells, it is assumed as a determining factor for cellular reactions against chemical drugs during treatment. There are multiple reports about the loss of potential for proliferation and differentiation and loss of osseous tissue with age $(12,13)$. Although age-dependent changes in MSCs of bone marrow have been a challenge in treatment processes, contradictory results have been obtained concerning the variations in the number of these cells with increase in age (14, 15). A limited number of reports have already pointed out to the reduction in fitness and efficiency of the aged MSCs, which can be due to the oxidative stress as ROS level, P53, and P21 increase $(13,14,16)$. Due to the fact that age-related changes in MSCs of the bone marrow are not clearly identified, reaction of these host cells to chemotherapy in different ages is still unclear. Therefore, due to the high penetration of doxorubicin into bone marrow and its adverse effects on MSCs, in the present study the biological changes and the incidence rate of cytotoxic effects of doxorubicin on the extracted MSCs from mice with different ages were examined.
This study was conducted in the Umbilical Cord and Stem Cells Department of Iranian Blood transfusion Organization and in Toxicology and Animal Poisoning Research Center of University of Tehran.

\section{Methods}

\section{Growth media and chemicals}

Cellular growth media of DMEM (Dulbecco's modified Eagle's medium; 1 gram per liter of D-glucose), adipogenesis differentiation medium, and osteogenesis differentiation medium were purchased form Gibco Company (USA) and were then supplemented with penicillin antibiotic (50 units per $\mathrm{mL}$ ), fungicide amphotericin B $(2.5 \mu \mathrm{g}$ per $\mathrm{mL}$ ), essential amino acids, and L-glutamine. Doxorubicin drug was purchased from Sigma Company. A solution of $1000 \mu \mathrm{M}$ in phosphate buffer saline (PBS) was prepared, and the stock solution was kept at $-20^{\circ} \mathrm{C}$. Alizarin Red, Oil Red O, and propidium iodide (PI) were obtained from Sigma Company (USA). Fetal calf serum (FCS) and trypsin/EDTA $(0.5 \%)$ were purchased from Gibco Company (USA), and collagen was supplied from STEM CELL Technologies (Canada). Monoclonal antibodies against surface markers were also provided from Dako Company (Denmark). The measurement kit of cytotoxicity detection for measuring lactate dehydrogenase (LDH) activity was purchased from Roche (Germany), and the solvents were supplied from Merk (Germany).

\section{Extraction of mesenchymal stem cells and cellular cul-} ture

Female BALB/c mice aged 1, 8, and 16 months (3 mice in each age group) were purchased from Razi Vaccine and Serum Research Institute (Iran). First, bone marrow aspiration of femur and tibia was done on the mice. The aspirated cells were kept in supplemented DMEM and 10\% FCS in an incubator at $37^{\circ} \mathrm{C}$ with $95 \%$ moisture and $5 \%$ $\mathrm{CO}^{2}$. After about 7 to 11 days, the mesenchymal stem cells colony (semi-fibroblast) was observed in the bottom of the cellular culture flask. After sequential cellular passages and removing non-adhesive cells with each time of replacing the cellular growth media, the mesenchymal stem cells were finally extracted in a period between 40 to 48 days (depending on the age of cells) (17).

After extraction, the cells were cultured in a tissue culture flask $\left(25 / 75 \mathrm{~cm}^{2}\right)$ in DMEM, containing 10\% FCS and $1 \%$ antibiotic/fungicide in an incubator at $37^{\circ} \mathrm{C}$ with 95\% moisture and $5 \% \mathrm{CO}_{2}$. The growth media was replaced every 3 days, and the cells were passaged using trypsin/EDTA enzyme.

In different test stages, the cells were counted using cell counter device (Sysmex XS 800i). In brief, $100 \mu \mathrm{L}$ of cellular suspension was mixed with $900 \mu \mathrm{L}$ of PBS, and the cells were counted in capillary mode. Finally, number of cells was calculated using the following formula. The obtained mean showed the number of cells in $1 \mathrm{~mL}$ of cell suspension.

Nimber of ceils $=$ Mean $\times$ Ditution factor $\times$ Volume factor 7

Growth rate in different ages of MSCs (the fourth cellular passage in logarithmic phase) has been obtained using 
the following formula:

$$
\mathrm{DT}=\mathrm{T} \ln 2 / \ln (\mathrm{Xe} / \mathrm{Xb})
$$

In this formula, DT is the doubling time of cells, $\mathrm{T}$ is the time of incubation, $X_{b}$ is the number of cells at the start of incubation, and $X_{e}$ is the number of cells at the end of incubation (18).

\section{Characterization of mesenchymal stem cells (surface staining)}

Morphological characteristics of the cells were investigated using invert microscope. Then, the cells (in the fourth passage after differentiation) were investigated in the presence of specific surface markers. To this end, cellular suspension was prepared in 5 microtubes $\left(10^{5}\right.$ cells in each microtube), moreover, and $5 \mu \mathrm{L}$ of the monoclonal antibodies CD34-PE, CD45- FITC, CD73-PE, CD105FITC and CD90-FITC were added to the cellular suspension. The samples were kept in the dark for 30 minutes at $4^{\circ} \mathrm{C}$. The cells were washed with $500 \mu \mathrm{L}$ of PBS, and were then fixed with $50 \mu \mathrm{L}$ of paraformaldehyde $1 \%$. Finally, analysis of the samples in comparison with the control group was performed using the flow cytometry device (Partec-PAS III) and FlowJo software $(19,20)$.

\section{Characterization of mesenchymal stem cells (In vitro differentiation)}

To examine the differentiation capability of MSCs, the bottoms of the wells in a 6-well plate were coated with collagen $4 \%$, and $2 \times 10^{5}$ cells (in the fourth cell passage) were cultured in each well. To conduct cellular differentiation into adipocyte and osteocyte lines, the cells were reserved for 21 days in specific growth media of adipo- genesis differentiation medium and osteogenesis differentiation medium, respectively (21).

A) Evaluation of differentiation into adipocyte cell line

The 0.18 grams of Oil Red O powder were solved in 50 $\mu \mathrm{L}$ of isopropanol $60 \%$, and the solution was filtered. To perform dyeing, first, the cells were fixed with ethanol $70 \%$ for 20 minutes. After washing, isopropanol $60 \%$ solution was added to each well for 5 minutes. Dyeing was performed with Oil Red O for 15 minutes and hematoxylin for 1 minute at room temperature. After final washing, the dye reaction of the cells was evaluated using reverse microscope (22).

B) Evaluation of differentiation into osteocyte cell line

The 1 gram of Alizarian Red powder was solved in 50 $\mu \mathrm{L}$ of distilled water. The solution was filterd and $\mathrm{pH}$ range was set to about 4.2. For dyeing, the cells were first fixed with ethanol $70 \%$ for 20 minutes, and isopropanol $60 \%$ solution was added for 5 minutes to each well after washing. Dyeing with Alizarian Red was performed in the dark for 45 minutes. After final washing, the dye reaction of the cells was evaluated using reverse microscope (22).

\section{Cytotoxicity assay (flow cytometry technique)}

To examine cytotoxicity and determine sublethal dose of the drug at each age, flow cytometry technique was employed after dyeing with propidium iodide (PI). The cells at each age were treated with different concentrations of doxorubicin drug $(25,50,100,200,400,800$, and 1200 $\mathrm{nM}$ ), and cellular death rate was estimated in comparison with the control group after 24 hours. An amount of $10 \mu \mathrm{L}$ of the PI dye was added to each cellular sample $\left(10^{6}\right.$ cells in each tube), and the samples were placed in the dark for 1 hour at $4^{\circ} \mathrm{C}$. The untreated sample was considered as
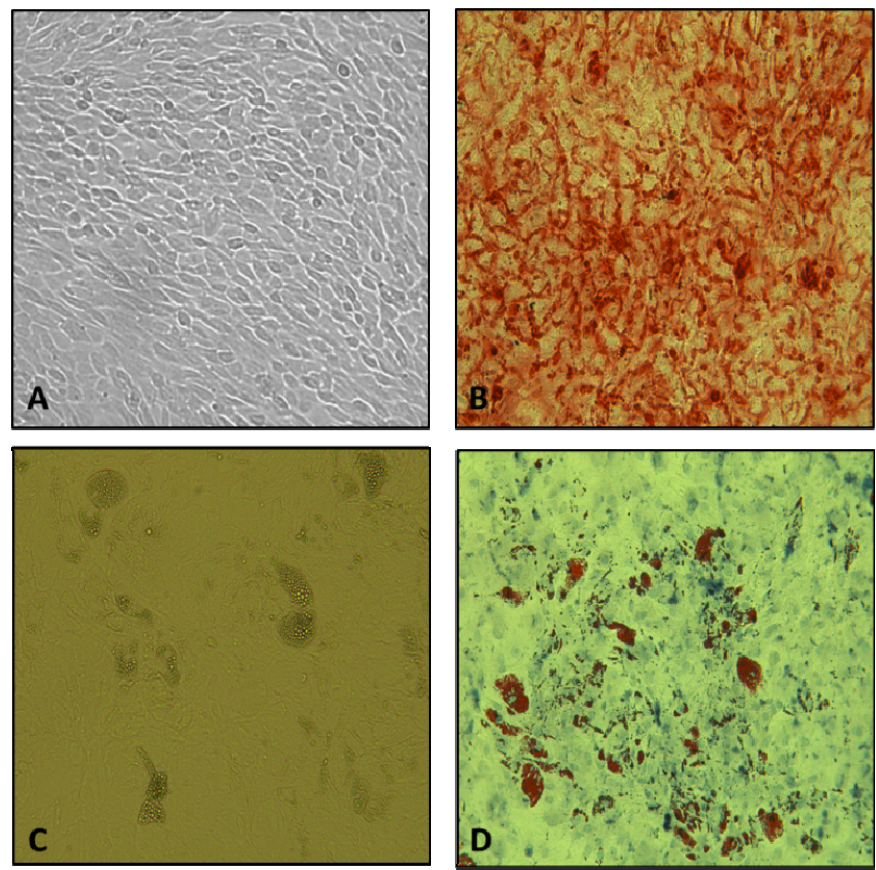

Fig. 1. MSCs differentiation into osteocyte (A and B) and adipocyte (C and D) cell lines. (A) Unstained sample of Alizarian Red dyeing, (B) Alizarian Red dyeing, (C) Unstained sample of Oil Red O dyeing, and (D) Oil Red Odyeing (magnification $\times 40$ ). 


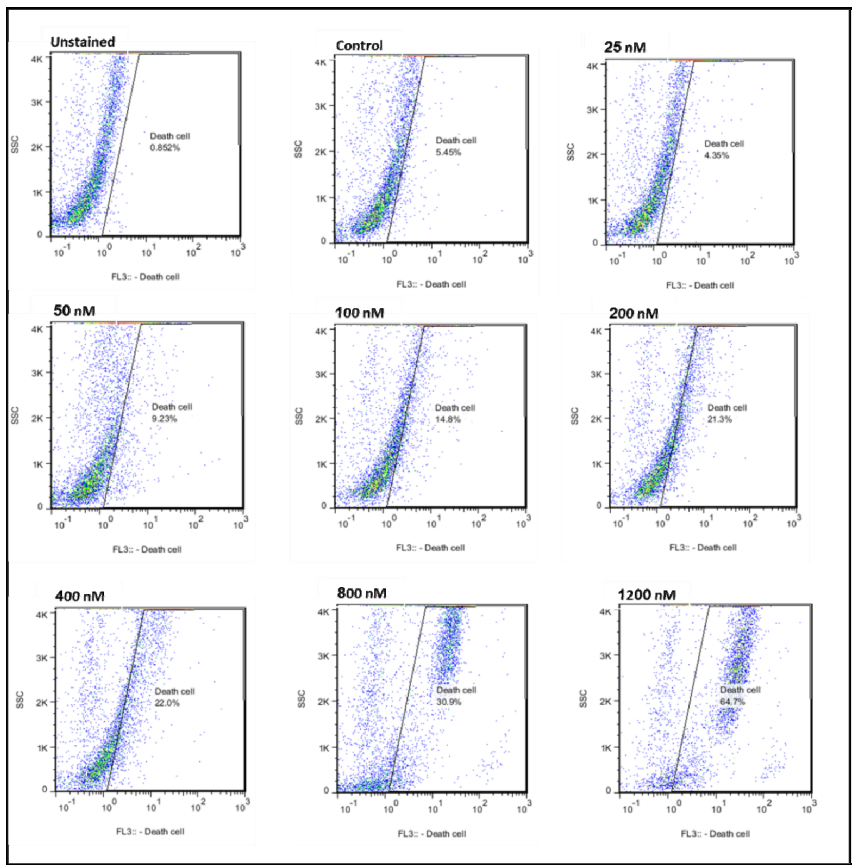

Fig. 2. Analysis of results from flow cytometry graphs related to the effect of different concentrations of doxorubicin drug on extracted MSCs from 8-month-old mouse.

control. Finally, the samples were analyzed using flow cytometry device (Partec-PAS III) and FlowJo software.

\section{Cytotoxicity assay (LDH test)}

To examine cell membrane health, LDH enzyme activity in the growth medium was estimated. This estimation was performed in accordance with the protocol provided by the manufacturer of the kit (Roche, Germany).

\section{Data analysis}

The results of the 3 iterations of the test were reported in the form of mean \pm standard deviation. The data obtained from cytotoxicity assays were compared using $t$ test with the significance level of $95 \%$.

\section{Results}

To examine cellular characteristics, MSCs capability for differentiation into the adipocyte and osteocyte lines was taken into account. To do so, the dyeing methods of Alizarian Red and Oil Red $\mathrm{O}$ were used, respectively. The results corroborated the capability of the MSCs separated from the bone marrow for differentiation into bone and fat cells (Fig. 1). In immunophenotyping method, using a flow cytometry device, over $85 \%$ of the mesenchymal stem cells extracted from bone marrows of the mice at different ages were positive for the presence of markers CD73, CD90, and CD105. Besides, more than $95 \%$ of the cells did not have the markers CD34 and CD45 on their surface. Results of immunophenotyping and cellular dif- ferentiation confirmed the removal of hematopoietic cells and good quality of MSCs differentiation. Moreover, the average of growth rate in logarithmic phase had a reverse relationship with the increase in age, so that DT in mice aged 1,8 , and 16 months were calculated as $29.8 \pm 2.06$, $35.1 \pm 2.5$, and $38.3 \pm 1.7$ hours.

Doxorubicin toxicity potential and $\mathrm{LDH}$ release were investigated using flow cytometry technique by PI dye. The results of the 2 tests were in considerable agreement and indicated that cytotoxic effect of the mentioned drug on MSCs at our considered ages increased dosedependence. Within 24 hours of exposure, effect of different concentrations of doxorubicin $(25,50,100,200,400$, 800 , and $1200 \mathrm{nM}$ ) on MSCs proliferation was investigated (Figs. 2, 3). IC50 results obtained from the flow cytometry test for the mice at different ages revealed that MSCs separated from 16-month-old mice had the most sensitivity to doxorubicin $($ IC50 $=861.43 \pm 10.06 n M)$, while the 1-month-old and 8-month-old cells came out second $\quad(I C 50=973.14 \pm 17.10 \mathrm{nM})$ and third $(I C 50=1036.61 \pm 24.42 \pi M)$, respectively. Besides, IC50 results obtained from LDH test for the mice aged 1, 8 , and 16 months were reported to be $970.80 \pm 23.72 n M, \quad 1047.19 \pm 21.30 \mathrm{nM}, \quad$ and $852.55 \pm 16.33 n M$, respectively. The dose with $25 \mathrm{nM}$ of the drug had no cytotoxic effect on the separated cells from any of the mice $(p<0.05)$, and therefore this dose could be considered as the shared sublethal dose for the 3 age groups. 
Through microscopic observations, cell deathdependent morphological changes at doses of 800 and $1200 \mathrm{nM}$ were clearly seen at different ages. These changes were maximally observed in cells exposed to a dose of $1200 \mathrm{nM}$ (Fig. 4). Cell death signs and symptoms included cytoplasm shrinkage, decrease in cell volume, nuclease fragmentation, granulation of cells, chromatin condensation, cytoplasmic vacuolation, and decrease in intercellular communication. Morphological changes of the differentiated viable cells were more in the age groups under treatment than the control group.

\section{Discussion}

Many of the drugs used for cancer treatment have adverse side effects (4). Effectiveness, side effects, and appropriate dose of a drug can be demonstrated by determining the effective and stable concentration of the drug in vitro and then in treated patients (23). Local destruction of the stem cells and bone marrow exhaustion are among the limitations of the clinical uses of chemical drugs against cancerous cells. Therefore, due to the roles of MSCs in biological processes, disturbance in the repair mechanism and negative effects on different cells after chemotherapy

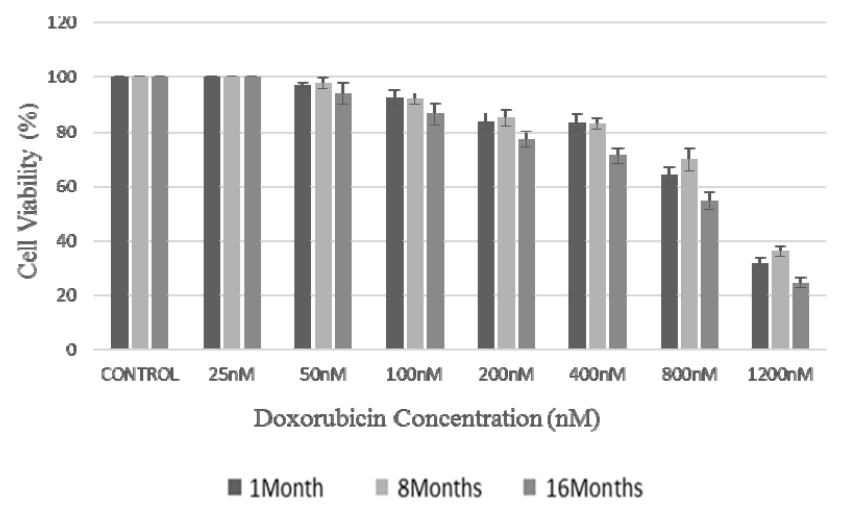

Fig. 3. Comparison of the viability (\%) of extracted MSCs from the bone marrow of BALB/c mice at different ages of 1,8 , and 16 months after encounter with different concentrations of doxorubicin; The obtained values were converted into the percentage of the control (taking untreated cells as $100 \%$ ).

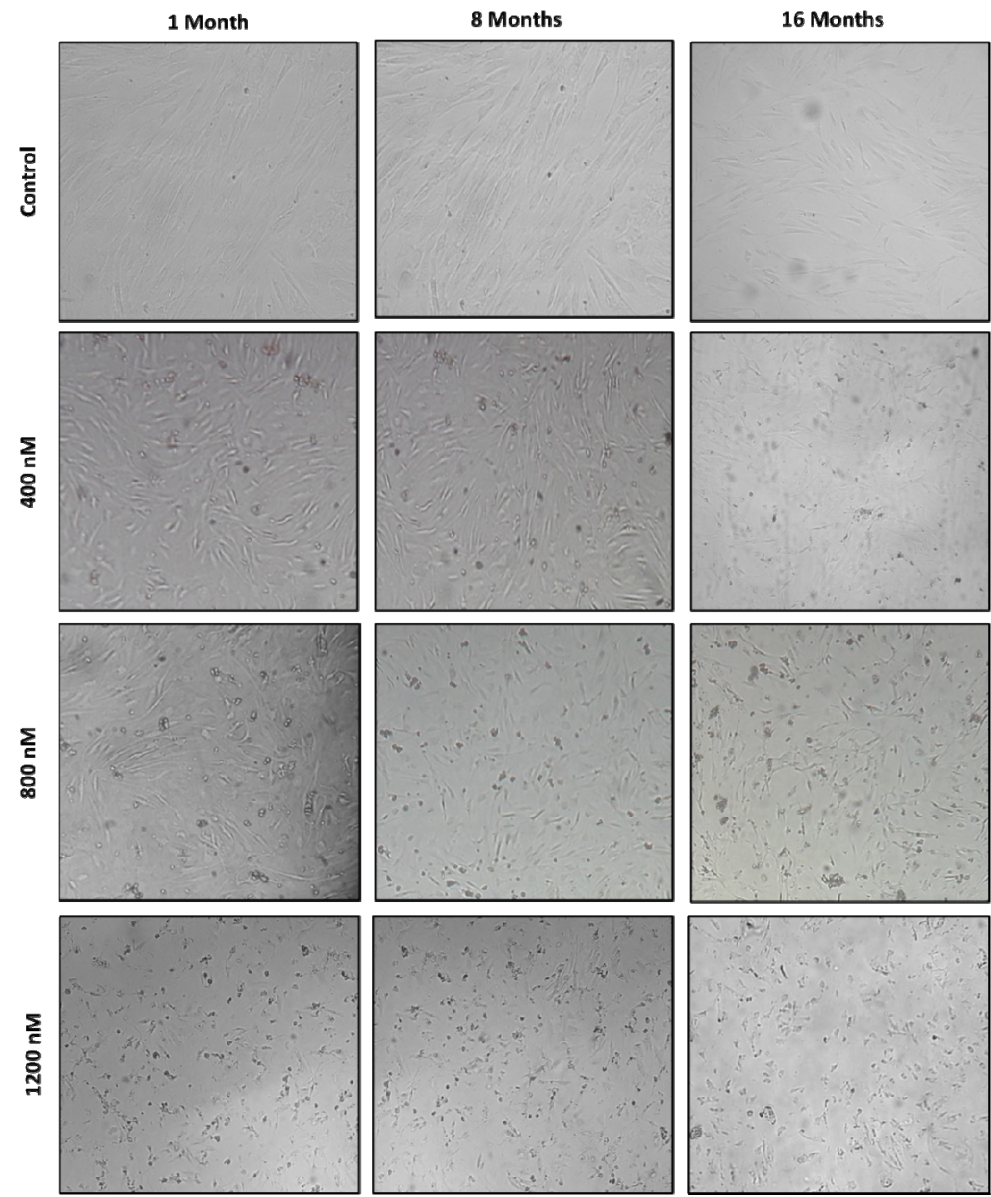

Fig. 4. Cell death-dependent morphological changes in MSCs extracted from the bone marrow of BALB/c mice at different ages of 1,8 , and 16 months after encounter with doxorubicin concentrations of 400, 800, and $1200 \mathrm{nM}$ (magnification $\times 100)$ 
can be expected $(5,6)$.

It has been reported that MSCs in rats can be doubled for about 22 times in 30 days in a way that the average time of each cell doubling is about 32 to 33 hours (11). In this study, due to age differences, a time span between 29 and 38 hours has been obtained in which the numbers of mice cells were doubled. In another study, the time of doubling for BM-MSCs in sheep was reported to be approximately 31.3 hours (24).

Based on the criteria provided by the International Society for Cellular Therapy (ISCT), MSCs, in addition to being capable of differentiation and of adhesion to plastic, must be positive in markers CD73, CD90, and CD105, whereas surficial molecules such as CD14, CD34, and CD54 are assumed to be negative to ensure noncontamination with hematopoietic cells (25). According to Baustian et al., expression of CD73 in cellular culture samples has increased with time passage, and thus it may rarely be observed in newer samples. However, CD90 can be used as a reliable marker for differentiation of the cells (26). Furthermore, although the surficial molecules were not reevaluated after treatment, the surviving stem cells still retain their surface characteristics in encounter with doxorubicin, as suggested in the literature (2). In the present study, the described markers by ISCT were used to detect the characteristics of the cells.

Doxorubicin is widely used in the treatment of solid tumors and blood malignancies $(3,27)$. As doxorubicin is lipophilic, inactive drug delivery to cell and penetration into nucleus are easily achieved (4). Antitumor drugs of anthracyclines group can exert their adverse effects through interfering into the function of enzymes involved in DNA replication. Their current mechanisms for causing toxicity include inhibition of topoisomerases I and II, excessive synthesis of ceramide, oxidative stress, and injury to DNA $(23,28)$. Another mechanism for causing toxic effects is DNA rotation due to instability of nucleosome $(27,29)$.

With regard to the side effects of different concentrations of doxorubicin, numerous studies have already been conducted to determine the sublethal dose of the drug ( 7 , $30)$. In this context, depending on the type and condition of the studies, different doses have been proposed as IC50 of the aforesaid drug $(31,32)$. In these studies, a wide range of doses ( 25 to $500 \mathrm{nM}$ ) have been proposed as the required concentrations for producing cytotoxic effects such as apoptosis in cardiomyocytes, genotoxic effects such as injury to the histone, and/or formation of free radicals in different cells $(27,31)$. In a study on doxorubicin effects, the sublethal dose of the drug $(10 \mathrm{nM})$ caused injury to DNA and permanently reduced telomere length on $\mathrm{mMSC}(7)$. In the present study, a doxorubicin concentration of $25 \mathrm{nM}$ has been calculated to be the sublethal dose. The non-agreement of our proposed dose with previous studies can be explained by the differences in the origin of cells, dissimilar conditions of cell culture and treatment, drug preparation method, and different clinical conditions. In our microscopic investigations, especially at low doses, more differentiated cells than the control ones were observed. This phenomenon occurs in cases of in- ducing cellular differentiation in the cells treatment with concentrations of doxorubicin lower than $1 \mu \mathrm{M}$ (23).

Intensity of the side effects arising from treatment with doxorubicin depends on the dose of the drug and repair capacity of the bone marrow of the patient (4). Unfortunately, in previous works, as the age ranges of the groups under study were not clear, contradictory results have been obtained concerning the proper dose for treatment (14). Consequently, determining the exact dose of the drug, with respect to age-dependent individual sensitivities, may result in a decrease in the drug side effects. Here, the most resistance to doxorubicin drug was observed in the MSCs extracted from bone marrows of the middle-aged mice. Difference in the sensitivity of different ages can be ascribed to the change of function and physiological processes with the increase in age. The higher sensitivity in old ages can be due to the decrease in fitness and physiological function and increase in proapoptotic factors, oxidative injuries, and proteins such as P21 and P53 $(12,14,33)$. Based on our obtained results, the higher metabolic activity of the young mice and their higher susceptibility by further encounters with free radicals (the mechanism of action of the drug), can be proposed as the cause of the more sensitivity of the young mice than the middle-aged ones $(34,35)$. Different studies have pointed to the reduction and/or unchanging hMSCs amount with the increase in age. In addition, numerous reports have already been provided concerning the reduction in differentiation potential, reduction in proliferation, and physiological deterioration with age (36-38). Agedependent changes in mMSC are much similar to those in hMSC in in vivo and in vitro conditions. Similarly, in rats, a reduction has been observed in the number of MSCs; however, contradictory results have been provided for BALB/c mice $(13,15)$. These variations can be ascribed to the differences in the age groups of the used animals, different places of extracting cells, different test methods, culture conditions, and varied physiological and metabolic conditions of animal bodies (14). For example, BALB/c mice are more sensitive to age-dependent changes than the other mice $(13,39)$. Moreover, adult $\mathrm{BALB} / \mathrm{c}$ mice were reported to have a reduction in the proliferation of the progenitor cells, while retaining many of their functioning and phenotypic characteristics under in vitro conditions (36). Kahn et al. stated that the number of live cells decreases in old and female BALB/c mice (40).

\section{Conclusion}

Determining appropriate concentration of the drug with fewer side effects on the normal cells in the body is of prime importance in cancer therapy; and this can be achieved by expanding the statistical population using a more varied age range, and paying attention to other individual characteristics.

\section{Acknowledgments}

This work was supported by Council for Stem Cell Sciences and Technology (Iran), Toxicology and Animal Poisoning Research Center, University of Tehran (Iran) 
and Iranian National Science Foundation.

\section{Conflict of Interests}

The authors declare that they have no competing interests.

\section{References}

1. Cruet-Hennequart S, Prendergast ÁM, Shaw G, Barry FP, Carty MP. Doxorubicin induces the DNA damage response in cultured human mesenchymal stem cells. Int J Hematol. 2012;96(5):649-56.

2. Oliveira MS, Carvalho JL, Campos ACDA, Gomes DA, de Goes AM, Melo MM. Doxorubicin has in vivo toxicological effects on ex vivo cultured mesenchymal stem cells. Toxicol Lett. 2014;224(3):380-6.

3. Doroshow JH, Synold TW, Somlo G, Akman SA, Gajewski E. Oxidative DNA base modifications in peripheral blood mononuclear cells of patients treated with high-dose infusional doxorubicin. Blood. 2001;97(9):2839-45.

4. Tacar O, Sriamornsak P, Dass CR. Doxorubicin: an update on anticancer molecular action, toxicity and novel drug delivery systems. J Pharm Pharmacol. 2013;65(2):157-70.

5. Gibaud S, Andreux J, Weingarten C, Renard M, Couvreur P. Increased bone marrow toxicity of doxorubicin bound to nanoparticles. Eur $\mathrm{J}$ Cancer. 1994;30(6):820-6.

6. Masse A, Ramirez L, Bindoula G, Grillon C, Wdzieczak-Bakala J, Raddassi K, et al. The tetrapeptide acetyl-N-Ser-Asp-Lys-Pro (Goralatide) protects from doxorubicin-induced toxicity: improvement in mice survival and protection of bone marrow stem cells and progenitors. Blood. 1998;91(2):441-9.

7. Buttiglieri S, Ruella M, Risso A, Spatola T, Silengo L, Avvedimento $\mathrm{EV}$, et al. The aging effect of chemotherapy on cultured human mesenchymal stem cells. Exp Hematol. 2011;39(12):1171-81.

8. Caplan AI. Adult mesenchymal stem cells for tissue engineering versus regenerative medicine. J Cell Physiol. 2007;213(2):341-7.

9. Uccelli A, Moretta L, Pistoia V. Mesenchymal stem cells in health and disease. Nat Rev Immunol. 2008;8(9):726-36.

10. Bosco DB, Kenworthy R, Zorio DA, Sang QXA. Human mesenchymal stem cells are resistant to Paclitaxel by adopting a nonproliferative fibroblastic state. PloS one. 2015;10(6):e0128511.

11. Liu Y, Song J, Liu W, Wan Y, Chen X, Hu C. Growth and differentiation of rat bone marrow stromal cells: does 5-azacytidine trigger their cardiomyogenic differentiation? Cardiovasc Res. 2003;58(2):460-8.

12. Zhou S, Greenberger JS, Epperly MW, Goff JP, Adler C, LeBoff $\mathrm{MS}$, et al. Age-related intrinsic changes in human bone-marrow-derived mesenchymal stem cells and their differentiation to osteoblasts. Aging cell. 2008;7(3):335-43.

13. Bellantuono I, Aldahmash A, Kassem M. Aging of marrow stromal (skeletal) stem cells and their contribution to age-related bone loss. Biochim biophys acta Mol basis dis. 2009;1792(4):364-70.

14. Stolzing A, Jones E, McGonagle D, Scutt A. Age-related changes in human bone marrow-derived mesenchymal stem cells: consequences for cell therapies. Mech Ageing Dev. 2008;129(3):163-73.

15. Wilson A, Shehadeh LA, Yu H, Webster KA. Age-related molecular genetic changes of murine bone marrow mesenchymal stem cells. BMC genomics. 2010;11(1):229.

16. Fehrer $\mathrm{C}$, Laschober $\mathrm{G}$, Lepperdinger G. Aging of murine mesenchymal stem cells. Ann N Y Acad Sci. 2006;1067(1):235-42.

17. Soleimani M, Nadri S. A protocol for isolation and culture of mesenchymal stem cells from mouse bone marrow. Nat Protoc. 2009;4(1):102-6.

18. Guide AACC. Tips and Techniques for Continuous Cell Lines, Jan. 2012. Manassas, VA.

19. Conget PA, Minguell JJ. Phenotypical and functional properties of human bone marrow mesenchymal progenitor cells. J Cell Physiol. 1999;181(1):67-73.

20. Pittenger MF, Mackay AM, Beck SC, Jaiswal RK, Douglas R, Mosca JD, et al. Multilineage potential of adult human mesenchymal stem cells. Sci. 1999;284(5411):143-7.

21. Ciuffreda MC, Malpasso G, Musarò P, Turco V, Gnecchi M. Protocols for in vitro Differentiation of Human Mesenchymal Stem Cells into Osteogenic, Chondrogenic and Adipogenic Lineages. Mesenchymal Stem Cells: Methods and Protocols. 2016:149-58.
22. Meirelles LdS, Nardi NB. Murine marrow-derived mesenchymal stem cell: isolation, in vitro expansion, and characterization. $\mathrm{Br} \mathrm{J}$ Haematol. 2003;123(4):702-11.

23. Gewirtz D. A critical evaluation of the mechanisms of action proposed for the antitumor effects of the anthracycline antibiotics adriamycin and daunorubicin. Biochem Pharmacol. 1999;57(7):727-41. 24. Heidari B, Shirazi A, Akhondi MM, Hassanpour H, Behzadi B, Naderi MM, et al. Comparison of proliferative and multilineage differentiation potential of sheep mesenchymal stem cells derived from bone marrow, liver, and adipose tissue. Avicenna J Med Biotechnol. 2013;5(2):104-17.

25. Dominici M, Le Blanc K, Mueller I, Slaper-Cortenbach I, Marini F, Krause D, et al. Minimal criteria for defining multipotent mesenchymal stromal cells. The International Society for Cellular Therapy position statement. Cytotherapy. 2006;8(4):315-7.

26. Baustian C, Hanley S, Ceredig R. Isolation, selection and culture methods to enhance clonogenicity of mouse bone marrow derived mesenchymal stromal cell precursors. Stem Cell Res Ther. 2015;6(1):151.

27. Yang F, Teves SS, Kemp CJ, Henikoff S. Doxorubicin, DNA torsion, and chromatin dynamics. Biochim biophys acta Rev Cancer. 2014;1845(1):84-9.

28. Foglesong PD, Reckord C, Swink S. Doxorubicin inhibits human DNA topoisomerase I. Cancer Chemother Pharmacol. 1992;30(2):123-5. 29. Yang F, Kemp CJ, Henikoff S. Doxorubicin enhances nucleosome turnover around promoters. Curr Biol. 2013;23(9):782-7.

30. Ciavarella S, Grisendi G, Dominici M, Tucci M, Brunetti O, Dammacco F, et al. In vitro anti-myeloma activity of TRAIL-expressing adipose-derived mesenchymal stem cells. $\mathrm{Br} \mathrm{J}$ Haematol. 2012;157(5):586-98.

31. Yang L, Wei L, Zhao W, Wang X, Zheng G, Zheng M, et al. Downregulation of osteopontin expression by RNA interference affects cell proliferation and chemotherapy sensitivity of breast cancer MDA-MB231 cells. Mol Med Rep. 2012;5(2):373-6

32. Fornari FA, Randolph JK, Yalowich JC, Ritke MK, Gewirtz DA. Interference by doxorubicin with DNA unwinding in MCF-7 breast tumor cells. Mol Pharmacol. 1994;45(4):649-56.

33. Stenderup K, Justesen J, Clausen C, Kassem M. Aging is associated with decreased maximal life span and accelerated senescence of bone marrow stromal cells. Bone. 2003;33(6):919-26.

34. Adelman R, Saul RL, Ames BN. Oxidative damage to DNA: relation to species metabolic rate and life span. Proc Natl Acad Sci. 1988;85(8):2706-8.

35. Frisard MI, Broussard A, Davies SS, Roberts LJ, Rood J, De Jonge $\mathrm{L}$, et al. Aging, resting metabolic rate, and oxidative damage: results from the Louisiana Healthy Aging Study. J Gerontol A Biol Sci Med. 2007;62(7):752-9.

36. Katsara O, Mahaira LG, Iliopoulou EG, Moustaki A, Antsaklis A, Loutradis D, et al. Effects of donor age, gender, and in vitro cellular aging on the phenotypic, functional, and molecular characteristics of mouse bone marrow-derived mesenchymal stem cells. Stem Cells Dev. 2011;20(9):1549-61.

37. Muschler GF, Nitto H, Boehm CA, Easley KA. Age-and gender-related changes in the cellularity of human bone marrow and the prevalence of osteoblastic progenitors. J Orthop Res. 2001;19(1):117-25. 38. Fehrer C, Lepperdinger G. Mesenchymal stem cell aging. Experimental gerontology. 2005;40(12):926-30.

39. Beamer W, Donahue L, Rosen C, Baylink D. Genetic variability in adult bone density among inbred strains of mice. Bone. 1996;18(5):397403 .

40. Kahn A, Gibbons R, Perkins S, Gazit D. Age-Related Bone Loss: A Hypothesis and Initial Assessment in Mice. Clin Orthop Relat Res. 1995;313:69-75. 